\title{
Guidelines for the multi-agency management of patients suspected or at risk of suffering from life-threatening abuse resulting in cyanotic-apnoeic episodes
}

Prepared by North Staffordshire Hospital Trust, Staffordshire Social Services and Staffordshire Police, and passed by Staffordshire Area Child Protection Committee, 12 January 1994. This summary of the full protocol prepared for the fournal of Medical Ethics by Professor David P Southall and Dr Martin P Samuels.

\section{Editor's note}

Following earlier discussion in the journal, various contributions were received, furthering the debate on covert video surveillance (CVS). The editor requested a full summary of the Staffordshire protocol after receiving $\mathrm{Mr}$ Thomas's critique of that protocol. He invited $\mathrm{Dr}$ Shinebourne, who was previously involved in the development of the techniques of CVS at the Royal Brompton Hospital, London, to comment on $\mathrm{Mr}$ Thomas's critique. Dr Evans and Professor Southall and Dr Samuels continue their debate on the issues.

\section{Introduction}

1.1 Children have the right to protection from abuse and ill-treatment. This protocol has been agreed between the agencies involved on behalf of Staffordshire's Area Child Protection Committee. It provides a framework for the investigation of suspicions of abuse among children referred to hospital following apparent life-threatening episodes for which there seems no other probable cause. The primary objective of any action by agencies in following this protocol is the welfare and safety of the child.

1.2 The procedures detailed in the protocol are designed to establish clearly whether there are grounds for concern about the child's care in a manner which provides safeguards from further possible harm. The protection of children from abuse demands good co-operation between the professionals involved, an open-minded response to concerns and a focus on the best interests of the child throughout any agency intervention. Once the facts have been established, and where grounds for concern about the child's care are substantiated, existing interagency procedures will be followed to ensure the continuing protection of the child.
1.3 This document was drawn up with particular reference to the establishment of guidelines for the management of life-threatening cyanoticapnoeic episodes in infants and young children where the mechanism is thought to involve intentional suffocation by a parent or carer.

\section{Principles of referral}

2.1 Where a paediatrician considers that cyanoticapnoeic episodes are possibly due to abuse by a parent or carer, he/she will refer to the child's local social services department and an initial strategy discussion will take place to consider how the investigation of concerns should proceed in the interests of protecting the child. The responsibility for organising this initial strategy discussion lies with the referring authority and should take place with minimal delay. The strategy discussion should discuss all available information and decide whether grounds exist for care proceedings to be instituted.

2.2 Following the initial strategy discussion, a decision to refer to North Staffordshire Hospital (NSH) may result. This may be because the child cannot be protected on the facts presented, or because there is a need for further medical investigations. Thus, paediatricians may refer a patient who has suffered from medically unexplained life-threatening cyanotic-apnoeic episodes to the Academic Department of Paediatrics for either further medical investigation, or covert video surveillance (CVS), or both.

2.3 At this stage, a second strategy discussion will be requested under Staffordshire Area Child Protection Committee Procedures, and this meeting will be convened in North 
Staffordshire. At this second strategy discussion, there will be discussion as to whether CVS, medical investigations or both are required.

2.4 Notes of any initial strategy discussion in the referring area will be made available to the second strategy discussion in North Staffordshire.

2.5 If the patient has already been transferred to NSH for a medical diagnosis and abuse is subsequently suspected, a single strategy meeting, combining the principles of the first and second strategy discussions, will take place in North Staffordshire with minimal delay.

\section{Strategy discussion/meeting before transfer to NSH}

3.1 Children suffering from respiratory difficulties including apparent life-threatening episodes (ALTE) are referred to the NSH from other parts of the country. In some cases, there may be concern that breathing difficulties have no apparent organic cause and suspicion exists that an adult may have attempted to suffocate the child. Where such suspicion exists, the multi-agency child protection procedures from within the child's area of origin should be initiated.

3.2 Working Together requires agencies who have a suspicion that a child is suffering significant harm to consult the investigating agencies (social services and/or police) and an initial strategy discussion should be convened to plan an investigation appropriate to the circumstances.

3.3 The complexity of cases wherein adults may be inducing a life-threatening episode of airway obstruction makes it essential that full consultation precedes any decision to investigate the circumstances using CVS or any other approach. Covert video surveillance could be used where it is agreed that evidence sufficient to ensure protection for the child through care proceedings is not already available from other sources. Legal advice is essential in reaching such a decision.

3.4 At the stage at which a paediatrician suspects that imposed upper airway obstruction might be responsible for ALTE, it is essential that adequate nursing supervision is provided for the child before and, depending on management, after, the initial strategy discussion has been convened, so that further, potentially dangerous abuse can be prevented. The child must be placed under a degree of nursing surveillance (ie, 'one-to-one' nursing care), which never allows the child to be alone in the care of the suspected parent.

3.5 If it is considered that a parent/carer may represent a risk to other patients/children on the ward, then sufficient supervision of other patients should be undertaken.

3.6 There are certain indicators which may suggest that the symptoms suffered by the child are induced by a parent:
(a) inconsistent histories from different observers

(b) symptoms and signs that are unusual or bizarre and inconsistent with known pathophysiology

(c) observations and investigations inconsistent with parental reports
(d) treatments which are ineffective or poorly tolerated

(e) symptoms and signs which begin only in the presence of one carer/parent

(f) parents who are unusually knowledgeable about the illness and its repercussions

(g) parents who contentedly fit in with ward life and attention from staff

(h) unusual or unexplained illness or death in previous children

(i) parents who have a history of unusual illness or themselves were abused as children

(j) parents who have a history of conduct or eating disorders (1).

3.7 Parents should NOT be made aware of the strategy discussion/meeting in advance. Failure to ensure this could prevent adequate protection for the child and might have serious consequences.

3.8 The initial strategy meeting/discussion will need to address the following issues to confirm or refute the diagnosis that the child's condition is being induced by a parent:

(a) clarification of history, obtaining details of any episodes that are reported to have occurred in the presence of a party other than the suspected perpetrator.

(b) check on temporal association between episodes beginning (rather than being witnessed) in the presence of one parent.

(c) check on details of personal, family and social history with other family members, social services and other health professionals - these details may be inconsistent, fabricated, or reveal that the parent has Munchausen's syndrome. Information will be needed regarding any previous social services or community health involvement, and any psychiatric history. Care should be taken in obtaining such information from health professionals with a responsibility for/to the suspected parent, so that the latter is not alerted to the enquiry at this stage.

(d) check on whether the parent has a relevant criminal record with local police and if any 
18 Symposium on covert video surveillance: Guidelines for the multi-agency management of patients suspected or at risk of suffering from life-threatening abuse resulting in cyanotic-apnoeic episodes

previous sudden death of a child in that family is known.

(e) If previous episodes of apnoea, cyanosis, cardiac or respiratory arrest, seizure or loss of consciousness have been recorded on multichannel physiological equipment, these would be examined to ascertain whether the pathophysiology present at the onset of and during episodes is typical of a natural or unnatural cause.

The meeting will consider what further investigation may be needed by relevant agencies, including the possible collection of additional physiological data. A decision to refer for a second strategy discussion at NSH should be undertaken where the assessment remains unclear or where there is insufficient evidence to ensure protection of the child at that point. Based on experience in previous cases, it is clear that the physiological data obtained during an episode of suffocation may not be conclusive evidence. Although physiological patterns may suggest suffocation, they are not universally considered diagnostic.

\section{Second strategy discussion or strategy meeting in North Staffordshire}

4.1 When an initial strategy discussion has resulted in a referral to NSH for consideration of CVS, the following will be invited to a second strategy discussion in North Staffordshire:

- Consultant paediatrician: Professor Southall or Dr Samuels

- Nurse manager (Child Protection) or deputy, NSH

- Business manager or deputy, NSH

- Staffordshire Social Services Child Protection Team

- Staffordshire Police Child Protection Team

- Legal representation from both the child's local authority and Staffordshire

- Representatives from the social services and police, the paediatrician and other health professionals from the area where the child normally resides.

Responsibility for inviting and co-ordinating this meeting is the responsibility of Staffordshire Social Services Department.

4.2 If a child is admitted to NSH without the prior convening of an initial strategy discussion in the area of referral (as described above), a strategy meeting must occur under Staffordshire Area Child Protection Committee Procedures. This situation could arise because the child was admitted to NSH for a medical diagnosis before suspicions arose. In addition to Staffordshire agencies, the following will be invited from the referring area: representatives from the Social Services Child Protection Team and a social services legal adviser, a police officer (child protection), medical and nursing staff from the referring area. The strategy meeting will be chaired by a senior manager from Staffordshire Social Services Department.

4.3 Agencies need to recognise the need for urgency in convening the strategy discussions/meeting. It is essential that parents are not alerted by any breach of confidentiality.

4.4 Where it is agreed that CVS should be used, the strategy discussion/meeting will identify and record through its chairperson, the lead agency or individual responsible for:

(a) discussion with parents regarding transfer to $\mathrm{NSH}$

(b) one-to-one nursing supervision of the patient prior to transfer to NSH

(c) notification to and briefing of paediatric ward staff and paediatric social worker at $\mathrm{NSH}$

(d) allocation of a Staffordshire child protection social worker and police officer

(e) organising trained nursing/medical staff to undertake surveillance

(f) dealing with the child in the event of deteco tion of the abuse

(g) planning for other children in the family io the event of detection of abuse

(h) immediate follow-up by agencies where the child lives, including a child protection conference

(i) obtaining psychiatric intervention/assessment of the parent following diagnosis

(j) review of the investigation

(k) seeking leave of the court for investigation at $\mathrm{NSH}$, if care proceedings are already in place

(1) action in the event of a parent/carer attempting to remove the child from the ward before an episode of abuse is identified.

\section{Optimal application of CVS}

5.1 NSH owns the video-recorders, TV monitors, etc. These are stored in a locked cupboard, the key being held by the clinical nurse specialist.

5.2 CVS should begin preferably early in the week and not at weekends. This allows the maximum number of working days to be available when adequate senior medical staff and ward staff are on duty.

5.3 Mode and process for briefing ward staff (both nursing and junior medical), nursing staff performing surveillance, police, and for specialist psychiatric consultation to perpetrator must be given careful consideration and discussed.

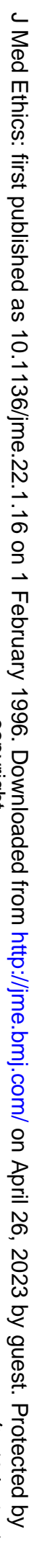


5.4 Child and parent must not move into the surveillance cubicle until all preparations required for CVS are completed.

5.5 Cameras must be positioned to allow observation of child at all times, but must not be invasive of the parents' privacy when he, she, or they are not handling the child or equipment. To ensure adequate observation and safety of the child, attention must be paid to:

(a) adequate lighting in the cubicle - this must be sufficient to ensure adequate vision is possible at all times, including at night

(b) the sound levels - this should be such that the child's vocalisations and monitoring equipment alarms may be heard. The television volume level must be limited internally to ensure this

(c) adequate communication systems between the observers of the video surveillance and ward staff - this will be principally by the use of a direct pager link between the observers and the nursing staff on the ward. This pager will have two levels of alert - high priority (ie intervene in cubicle immediately) and low priority (ie attend or telephone immediately surveillance office)

(d) access to the cubicle - this must at all times be kept clear to allow video surveillance staff a ready passage to the cubicle in the case of emergencies

(e) functioning of equipment - all equipment, including the pager system, will be checked by hospital electronics staff prior to commencement of surveillance, and the pager will need to be tested at the beginning of every nursing shift. A written record will be made of this check, and any malfunction should be repaired before operations commence.

5.6 The paediatrician will ensure that physiological monitoring equipment is attached to the child, as used for sleep studies, prior to or at the onset of CVS. This equipment will include a skin (transcutaneous) PO2 monitor as used for all infants and children in the NSH with cyanotic episodes, and will provide an effective, early alarm for major falls in blood oxygen levels. This alarm can be heard by nurses on the ward and by the surveillance nurses.

5.7 If the surveillance equipment fails, constant one-to-one nursing supervision of the patient will be provided whilst the equipment is repaired or replaced.

5.8 To provide continuous video surveillance, there will be two nursing staff (Registered mental nurse - RMN, Registered general nurse - RGN, Registered sick children's nurse RSCN) of F or G grades on duty at any one time who must undergo prior training (details of training available on request). Whenever possible, one member of the surveillance team should hold the RSCN qualification. Each pair of nurses will undertake eight-hourly shifts, with additional fifteen-minute hand-over periods. In some instances, it would be appropriate for medical staff to be present to aid interpretation of the parent/child interaction. Nursing staff on the ward who hold the RSCN qualification will also always be available.

5.9 One of the trained surveillance nursing staff will be engaged for no longer than a half-hour period observing the video monitor and logkeeping in order to minimise fatigue. The other will be present supporting his/her colleague and helping him/her interpret changes seen on the video screens. The surveillance staff will cover each other during meal breaks and other essential breaks, and these should be taken when surveillance is less critical (for example, when the suspected perpetrator is out of the cubicle, or when a ward nurse is in the cubicle performing observations or a procedure. The surveillance nurses will have received full instructions on when to alert the nurse in charge (via the radio-pager) and when to intervene in the cubicle (instructions available on request).

5.10 During CVS, two videotapes will be used simultaneously, with an overlap (of at least five minutes between the tapes). Tapes must be consecutively numbered, and recorded in the log kept by the surveillance nurses. The identity of the observer inserting the tapes will be indicated upon the log. All tapes must be labelled and kept in a safe place, and are the responsibility of the NSH. At the conclusion of recording, videotapes and logs will be stored by Staffordshire police, and available to social services in accordance with the recommended practice regarding the video-recording of children. When the proceedings have ended, the video-recordings will be returned to the Academic Department of Paediatrics, NSH. If no proceedings are being considered, then the tapes and $\log$ sheets will form part of the medical record and will be retained by the NSH (see paragraph 9.3).

5.11 Following an observed incident, police and a child protection social worker will be asked to attend. They will be responsible for proceeding with the investigation, including any discussion with the alleged perpetrator and for providing immediate protection for the child. The nurse in charge of the ward will ensure the cubicle is secured until advised by the police.

\section{Management of surveillance staff}

6.1 Two nurses per shift with $R M N, R G N$, or RSCN qualifications will be engaged especially 
20 Symposium on covert video surveillance: Guidelines for the multi-agency management of patients suspected or at risk of suffering from life-threatening abuse resulting in cyanotic-apnoeic episodes

to perform video surveillance. The nursing staff will be responsible to the nurse manager (child protection) and will undergo training in the use of video surveillance, log-keeping and use of all technical equipment prior to attending the hospital to perform surveillance. This training will include the viewing of videorecordings during suffocation obtained from previous cases.

6.2 When attending the hospital to begin surveillance, the nurses will be briefed and appropriately trained on the case by Professor Southall/Dr Samuels and the nurse manager (child protection) or clinical nurse specialist. This briefing will include a short synopsis of the clinical situation of the family.

6.3 The need for confidentiality will be stressed at the briefing, although confidentiality is implicit in their professional responsibilities.

6.4 The responsibilities of the nurses in relation to recording concise and accurate notes will be outlined, especially in relation to the suspected abuser's behaviour and interaction with the child, which will be taken to subsequent case conferences and may be used as evidence in any court case. The United Kingdom Central Council for Nursing, Midwifery and Health Visiting (UKCC) Professional Identification Number and expiry date will be kept by the nurse manager (child protection).

6.5 The observers must be given concise information on the circumstances under which they must alert the nurse in charge of the ward. This will include instruction on use of the high priority page alert to bring the ward nurse immediately into the child's cubicle in the event of actual or possible abuse, and the use of the low priority page alert to request the ward nurse to attend or contact immediately the surveillance nurses in the event of unexplained behaviour of the parent (for example manipulating the recording equipment or monitors).

6.6 The surveillance nurses must observe and record at all times the behaviour of the suspected abuser when interacting with the child.

6.7 Accurate notes must be made of the above during the full duration of surveillance. These must be written legibly on continuation sheets, consecutively numbered and provided by the hospital. Cross reference to the tape numbers must be made in the log. The surveillance nurses may be asked to explain the behaviour of the suspected abuser to the police. Each page should contain the child's name, hospital number, page number, date and time. In addition, all entries must be signed, using the full signature of the nurse.

6.8 On taking over the case the surveillance nurses must denote transfer of responsibility by assessing the current situation and recording clearly their findings.

6.9 If in the course of CVS, a surveillance nurse meets a situation which he/she feels is outside his/her competence to explain or interpret, he/she must immediately contact the nurse in charge of the ward for clarification (low priority page alert).

6.10 Although the surveillance nurses are not expected to participate in direct patient care, they may be required in an emergency to enter the cubicle and protect the child.

6.11 The surveillance nurses must be debriefed at the first available opportunity by the nurse manager (child protection) or clinical nurse specialist (see section 8 ).

\section{Discussion with other family members after abuse has occurred}

7.1 Senior medical or nursing staff on the ward may have initial contact and provide support for other family members, but should also refer them to the child protection social worker and police officer for information. This communication marks the beginning of further planning and must be sensitive and clear. Members oB the child's family may be in a state of shock and this will affect their reactions, including their ability to make decisions and plans for the future.

\section{Staff support and feedback}

8.1 Working with sick children carries a high degree of stress for staff from all disciplines, including the child protection team. If cases of child abuse, actual or potential, are added to this along with this particular system for identifying abuse cases, a scenario of extreme emotional stress can be present for staff of all disciplines and at all levels.

8.2 It is acknowledged that staff support must be addressed and will form part of the process of the investigation. It is part of the responsibility of senior medical and nursing staff and managers to try to ensure that an appropriate level of support is provided for staff under their supervision and that resources are dedicated to this.

8.3 In order to ensure understanding of the investigation and its results by all members of staff within all agencies, the professionals involved (medical, nursing, social services, police) will be invited to a 'debriefing' meeting as soon as possible after the abuse has been confirmed by CVS, and/or child protection procedures have been instituted. The debriefing will review procedures and make recommendations where necessary to improve practice and the protocol.

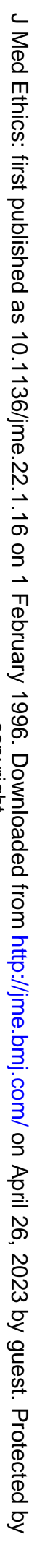




\section{Access to videotapes}

9.1 In the case where child abuse is diagnosed, and both criminal and civil proceedings follow, all the videotapes become the responsibility of the police after the abuse has been documented. The storage, access and disposal of these tapes will be as laid down by the Police and Criminal Evidence Act, and Codes of Practice.

9.2 In the case where child abuse is diagnosed, and in which the degree of abuse is considered sufficient for civil/care proceedings, but insufficient for criminal proceedings, all the videotapes will be stored in a safe and secure way by the social services department for the referring area.

9.3 In the case where no abuse is demonstrated during CVS, all videotapes will be the property of NSH and stored in a safe and secure way in the NSH. All tapes will be stored within a box sealed with tape in a locked cupboard. The box will be marked with the child's name, NSH record number, number of tapes, date of monitoring and who sealed the box. If tapes are removed for whatever purpose, a signed record of the following should be left within the box: the date the box is opened, re-sealed and by whom and the number of tapes removed.

9.4 Access to tapes should be kept to a minimum, consistent with the interest of the child and the interest of justice.

\section{Appendices 1-4 of the protocol}

Appendix 1 is a sample agenda for the multi-agency strategy discussion/meeting. This includes introduction, presentation of medical and social history, and current concerns, and a multi-agency discussion. If it is agreed that CVS is to be instituted, the issues listed in section 4.4 (a to l) are decided upon. If CVS is not the agreed option, the meeting considers actions to be taken by local professionals.

Appendix 2 outlines the training to be given to nursing staff involved in covert video surveillance. This includes a 3 to 4 -hour training session discussing relevant medical literature, and the guidelines provided by the Area Child Protection Committee, and observing video recordings from previous patients, showing some of the potential problems that may occur during surveillance. The nursing staff are individually assessed, firstly as to their agreement to undertake surveillance, and secondly to ensure that they are competent and understand their role. ${ }^{\star}$

Appendix 3 lists the instructions for the observers during CVS. It states that the absolute priority is protection of the child and although observers are observing for an attempt at suffocation, they must be aware of other potential harm to the child. The observers are expected to understand the role of surveillance, the technology involved, and to be aware of the clinical situation of the family. They are expected to observe the child at all times, keep accurate notes, and know when to contact the nurse in charge of the ward. Observing staff will take turns to be the main observer for no longer than half an hour. Two videotapes will record simultaneously. The observers will not be expected to participate in direct patient care. It is expected that at no time should the parent undergo CVS independently of the child. Instructions on when the observer is expected to intervene are given, and what action must be taken if the nurse in charge of the ward has not entered the cubicle within 25 seconds after a high priority alert. If abuse is identified, and the parent attempts to remove the child from the ward, the observer must give assistance to the ward staff to ensure the child is kept on the ward. If the parent attempts to remove the child from the cubicle before abuse has been observed, the high priority page must be activated. The nurse in charge of the ward will then decide what action is to be taken, in common with any other case of suspected child abuse. Such decisions will be made preferably in advance in consultation with medical staff. The nurse in charge of the ward should also be contacted in the case of any? uncertain behaviour of the parent. At all times confidentiality must be maintained.

Appendix 4 lists the names and contact numbers of agencies in North Staffordshire involved in CVS.

*The authors had previously stated their opinion that such surveillance activity should preferably be carried out by the police (2).

Professor David P Southall, MD, FRCP, is Professor of Paediatrics in the Academic Department of Paediatrics, University of Keele, North Staffordshire Hospital, Stokeon-Trent. Martin P Samuels, MD, MRCP, is Consultant Paediatrician in the same department.

\section{References}

(1) Samuels M P, Southall D P. Munchausen syndrome by proxy. British journal of hospital medicine 1992; 47: $759-762$.

(2) Southall D P, Samuels M P. Some ethical issues surrounding covert video surveillance - a response. fournal of medical ethics 1995; 21 : 104-105. 